\title{
EVALUATION OF CASPASE 9 EXPRESSION IN INDUCED HAMSTER BUCCAL POUCH CARCINOMA
}

\author{
Ahmed Abdel-Shakour Abdel-Hafeze*, Mohamed Mahmoud Ahmed** and Mohamed Gomaa Attia Zouair***
}

\begin{abstract}
The aim of the current study was to evaluate the expression of caspase 9 as an apoptotic marker in 7, 12- Dimethylbenz (a) anthracene (DMBA) induced hamster buccal pouch (s) (HBP(s)) carcinoma. Material and methods: The experimental animals used in the current study were fifty-five golden Syrian hamsters weighting 80-129 g, and obtained from the animal house, Cairo University. The experimental animals were divided into two main groups: Group A (normal group) 5 hamsters were taken, not treated and served as control. Group B: The remaining animals (50 hamsters) were treated with the same procedures: HBPs were painted for 18 weeks with DMBA, 3 times a week. Then, a direct lesional injection by different kinds of treatment: gold nanoparticles (AuNPs) (group B2), AuNPs +laser (group B3) cisplatin (group B4), AuNPs conjugated with cisplatin and laser irradiation (group B5), were subsequently employed. After that, various investigation: hematoxylin and eosin (H\&E) stain and immunohistochemical staining utilizing antibody against caspase 9 were done. Results: Gross observation revealed that there is a variation in the reduction of the distribution and size of tumors between the treated groups. Immunohistochemical results revealed that caspase 9 expression has a wide variability in the area percentage distribution through-out the treated groups. Conclusions: AuNPs in conjunction with cisplatin and visible laser irradiation have positive effect on tumor ablation. Moreover, intra-tumoral application of AuNPs in conjunction with cisplatin and visible laser irradiation is considered as a promising therapeutic agent in the regression of HBP DMBA induced carcinoma.
\end{abstract}

KEYWORDS: Squamous cell carcinoma, Apoptosis, Cisplatin, Gold nanoparticles

\section{INTRODUCTION}

Oral squamous cell carcinoma (OSCC) is one of the major global health problems with an annual estimated incidence of 300,000 newly diagnosed cases $^{(1)}$. 7, 12-Dimethylbenz (a)anthracene (DMBA) a polycyclic aromatic hydrocarbon, is widely employed to induce oral carcinoma in experimental animals including hamster buccal pouch (HBP) ${ }^{(2,3)}$. It has been found that DMBA induced oral tumors expressed biochemical and molecular characteristics similar to that of human oral tumors ${ }^{(4)}$. Among various factors, apoptosis was found to play a pivotal role in the regulation of OSCC ${ }^{(5)}$. Despite the significant advances made in conventional treatment for cancer such as surgery, radiotherapy, chemotherapy or combinations of them over the past decades, head and neck squamous cell carcinoma (HNSCC) continues to have a dismal prognosis, with a 5-years overall and disease-free survival of estimated $\sim 50 \%{ }^{(6)}$.

Cisplatin is one of the most effective anticancer drugs and is widely used for the treatment of various malignant tumors, including HNSCC ${ }^{(6-8)}$. Apoptotic cell death of tumor cells induced by cisplatin mediated mainly via the activation of various signal transduction pathways, including calcium and death receptor signaling, in addition to activation of mitochondrial pathways ${ }^{(9-11)}$. Among caspase proteins, cisplatin was found to induce apoptosis through caspase-9 activation ${ }^{(12,13)}$.

\footnotetext{
* Assistant lecturer, Oral and Dental Pathology Department, Faculty of Dental Medicine, Boys, Cairo, Al-Azhar University ** Professor, Oral and Dental Pathology Department, Faculty of Dental Medicine Boys, Cairo, Al-Azhar University, Egypt. *** Professor and Head of Oral and Dental Pathology Department, Faculty of Dental Medicine Boys, Cairo, Al-Azhar University, Egypt.
} 
Currently, cancer research has been focused on improving cancer diagnosis and treatment methods via new techniques, among which are using nanoscale particles ${ }^{(14)}$. It has been reported that there is a huge potential to use nanoparticles in cancer therapy. Nanotechnology involves the design, characterization, production and application of nanoscale structures, devices, and systems ${ }^{(15)}$. Interestingly, updating research has proved photothermal therapy (PTT) to be a successful method for treating superficially located HBP carcinomas ${ }^{(16)}$. Currently, the most advanced area of nanomedicine is the development and use of nanoparticles for drug delivery ${ }^{(17-19)}$.

\section{MATERIAL AND METHODS}

The experimental animals used in the current study were golden Syrian hamsters. The hamster was used as model for carcinoma induction utilizing DMBA as chemical carcinogen. Then, a direct inralesional injection by different kinds of treatment: AuNPs, cisplatin, AuNPs conjugated with cisplatin and laser irradiation, were employed. After that $\mathrm{H} \& \mathrm{E}$ stain and immunohistochemical staining utilizing caspase 9 were used.

\section{Carcinogen Preparation:}

DMBA was prepared by dissolving $0.5 \mathrm{gm}$ DMBA powder in $100 \mathrm{ml}$ paraffin oil (SigmaAldrich). Citrate capped-AuNPs preparation: The brand: (NanoTech Egypt for Photo-Electronics) AuNPs was synthesized as follow: citrate stabilized spherical AuNPs with an average diameter of $20 \mathrm{~nm}$ were synthesized using citrate reduction method. Cisplatin: Platinum coordination compound from Pfizer medical company. Cisplatin conjugated with nanogold: Cisplatin conjugated AuNPs was, initially, prepared following the same method as used in AuNPs preparation. The experimental animals were classified into two main groups: Group A (normal group): 5 hamsters were taken, not treated and served as control. Group
B: The remaining animals (50 hamsters) were treated with the same procedures in which the buccal pouches were painted for 18 weeks with DMBA, 3 times a week. Then the hamsters were randomly divided into the following five equal subgroups, 10 hamsterseach: Group B1 (DMBA treated group): at 18 weeks animals were grossly examined, euthanized and tissue sections were, then, obtained and prepared in order to examined histologically. Group B2 (DMBA- AuNPs treated group): The HBP lesions of this group were directly injected intra-tumoral with AuNPs $(10 \mathrm{mg} / \mathrm{kg})$ by insulin syringe. Group B3 (DMBA- AuNPs laser radiation treated group): The lesions of this group were directly injected with AuNPs $(10 \mathrm{mg} / \mathrm{kg})$ by insulin syringe. This step was repeated 3 times every 72 hours. Then, the animals were left unhanded for 4 weeks. Group B4 (DMBAcisplatin-laser radiation treated group): The tumors of this group were directly injected with cisplatin $(10 \mathrm{mg} / \mathrm{kg})$ by insulin syringe. Group B5 (DMBA- AuNPs conjugated with cisplatin laser radiation treated group): The lesions of this group were directly injected with AuNPs (10 mg/ $\mathrm{kg}$ ) conjugated with cisplatin by insulin syringe. The animals in groups B2, B4 and B5 were directly exposed to light emitting diode $(520-560 \mathrm{~nm})$ wave length $(150 \mathrm{mw}, 8 \mathrm{~mm}$ diameter) for 15 minutes within 2 minutes of injection to limit particle diffusion beyond the lesion boundaries. This step was repeated 3 times every 72 hours. Then, the animals were left unhanded for 4 weeks.

\section{Euthanization and investigations}

After 2 days of experiment, 2 animals from groups B2, B3 and B5 were grossly observed, prepared to be euthanized for tissue section preparation in order to examine the distribution of the particles material used in the tissue. The other 8 animals were left unhanded for 4 weeks until the end of the experiment. Tissue sections were prepared for H\&E stain and immunohistochemical staining utilizing antibody against caspase 9 . 
The degree of positive staining for antibody was evaluated by a well-established semi-quantitative scoring on a scale ranging from negative to strong positive staining as mentioned at table (1) ${ }^{(20)}$.

TABLE (1): Immunohistochemical staining scoring system.

\begin{tabular}{|c|c|}
\hline Area \% & Staining positivity \\
\hline $0-5 \%$ & Negative \\
\hline $5-25 \%$ & Week \\
\hline $25-50 \%$ & Moderate \\
\hline More than $50 \%$ & Strong \\
\hline
\end{tabular}

The image analysis was performed using Leica QWIN V3 image analyzer. This was done in the Oral and Dental Pathology Department, Faculty of Dental Medicine, Boys, Cairo, Al-Azhar University. The data obtained from computer image analysis were presented as mean way analysis of variance (ANOVA) test for statistical analysis of the difference between the groups used.

\section{RESULTS}

Gross observation: The right HBP mucosa of the animals in groups B1 (DMBA treated group), B2 (AuNPs treated group) and B4 (cisplatin + Laser treated group) showed ulcerative areas, multiple tumor masses and multiple nodular elevations. The right HBP mucosa of the animals in groups B3 (AuNPs + Laser treated group) and B5(cisplatin conjugated AuNPs + Laser treated group) showed noticeable reduction in distribution of tumor growth with regression in the tumor size compared to that seen in group B1 (Figs 1.A-D)

\section{Histopathological and immunohistochemical results}

Group A (normal group): Histological sections, using $H \& E$ stain revealed that the mucosa is composed of a thin stratified squamous cell layers, consists of two to four layers of squamous cells exhibiting slight keratinization. The immunohistochemical staining using caspase 9 exhibited mild positive cytoplasmic expression

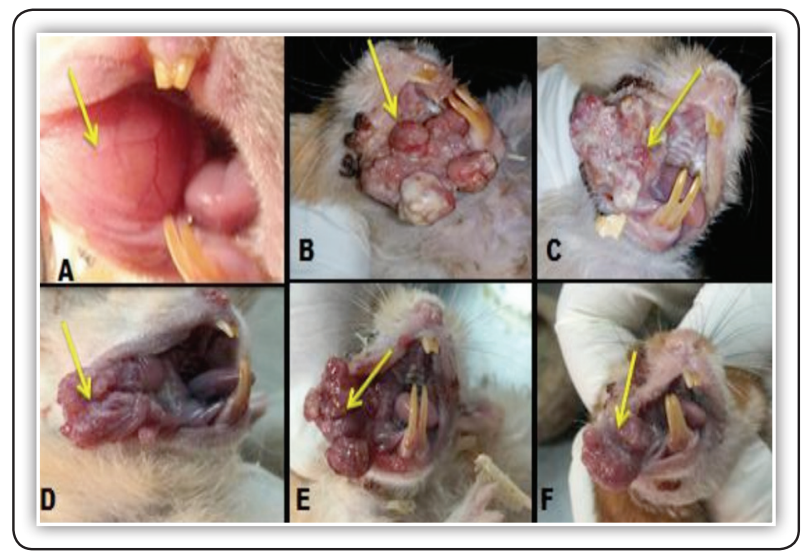

FIG. 1A; HBP mucosa in normal group showing pink and smooth surface mucosa. Fig.1B: HBP mucosa in B1 group showing multiple nodular elevations and ulcerative areas. Fig.1C: HBP mucosa in B2 group showing ulcerative areas and multiple white tumor masses. Fig.1D: HBP mucosa in B3 group showing ulcerative areas and multiple white tumor masses. Fig.1E: HBP mucosa in B4 group showing multiple nodular elevations and ulcerative areas. Fig.1F: HBP mucosa in B5 group showing reduction in tumor masses size.

(19.87\%) throughout the epithelial layers (Figs, 2 A\&B). Group B1 (DMBA treated group): $H \& E$ stain showed that the overlying epithelium has obvious dysplastic features in multiple areas and providing an evidence of prominent true invasion with formation of epithelial nests. Various appearances of SCC nests: well differentiated as well as moderately differentiated were observed. Invasion of the epithelial nests were seen. Caspase 9 exhibited negative cytoplasmic expression (4.38\%) in the invasive well differentiated SCC nests (Figs.2 C\&D). Group B2 (DMBA+ AuNPs treated group): $\mathrm{H} \& \mathrm{E}$ stain showed obvious presence of dysplastic features in multiple areas within the overlying epithelium cells. Invasion of malignant epithelial cells into the underlying connective tissue with keratin nests formation was seen. Invasion of the epithelial nests with the appearances of SCC nests: well differentiated as well as moderately differentiated was observed. Caspase 9 exhibited mild positive cytoplasmic expression (17.93\%) in the invasive well differentiated SCC nests (Figs. 3 A\&B). Group B3 (DMBA+ AuNPs 
+laser treated group): H\&E stain displayed that the epithelial cells in multiple areas exhibiting abundant features of malignancy, true invasion of well differentiated SCC. Caspase 9 exhibited moderate positive cytoplasmic expression (43.89\%) in the invasive SCC nests (Figs.3 C \& D). Group B4 (DMBA+ cisplatin +laser treated group): $\mathrm{H} \& \mathrm{E}$ stain displayed obvious dysplastic feature in multiple areas and true invasion with formation of epithelial and keratin nests. Appearances of SCC nests: well differentiated as well as moderately differentiated were observed. Caspase 9 exhibited mild positive cytoplasmic expression $(16.43 \%)$ in the invasive well differentiated SCC nests (Figs. 4 A\& B). Group B5 (DMBA+ AuNPs conjugated with cisplatin + laser group): H\&E stain revealed that the overlying epithelium cells have conspicuous dysplastic feature in multiple areas. A true invasion of well differentiated SCC was seen in the subepithelial C.T.Caspase 9 exhibited strong positive cytoplasmic expression (59.09\%) in the invasive SCC nests (Figs.4 C \& D).

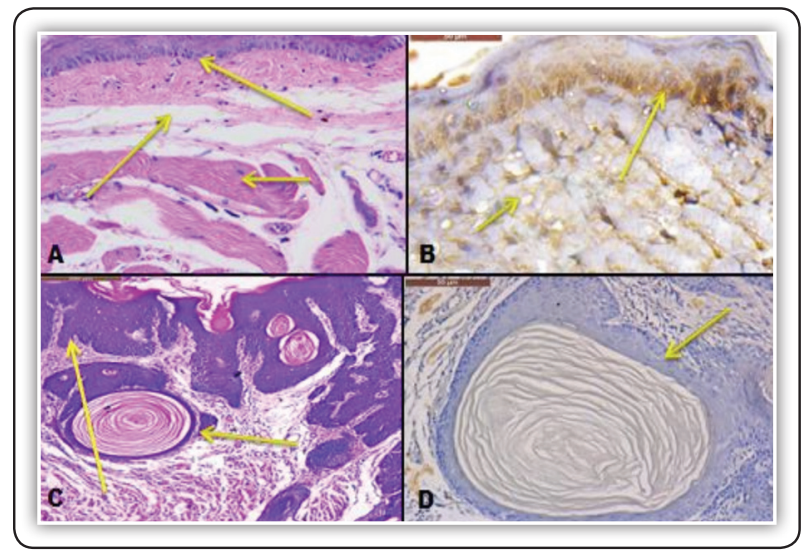

FIG.2A: H\&E stain for HBP mucosa in normal group showing epithelium layers with flattened rete ridges. Subepithelial connective tissue and muscular layers. Fig.2B: Expression of caspase 9 in normal animal group showing positive cytoplasmic staining throughout the epithelial layers. Connective tissue showing nonspecific reaction. Fig.2C: H\&E stain for HBP mucosa in DMBA treated group showing epithelium with dropshaped rete ridges. Notice, subepithelial invasion of well differentiated SCC nests. Fig.2-D: Expression of caspase 9 in DMBA treated group showing negative cytoplasmic staining in the cells of epithelial nest.

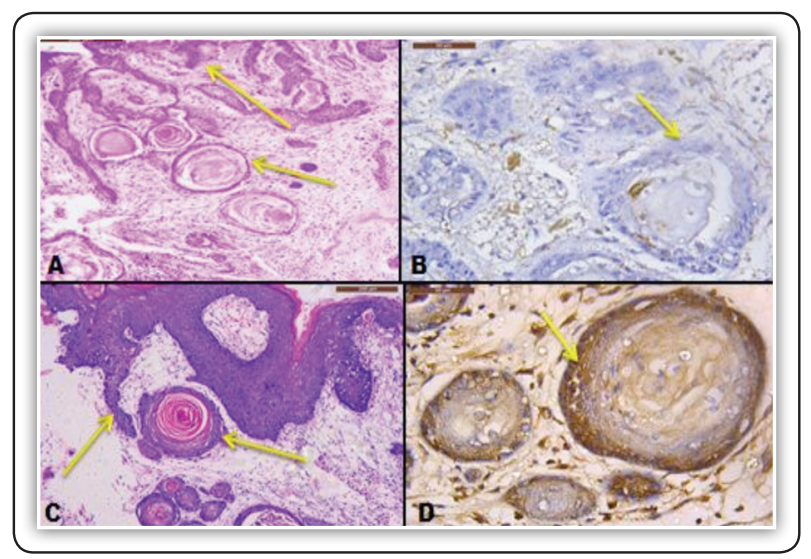

FIG.3A: H\&E stain for HBP mucosa in AuNPs treated group showing epithelium with drop-shaped rete ridges. Subepithelial invasion of well differentiated SCC nests. Fig.3B: Expression of caspase 9 in AuNPs treated group showing variable immunostaining include negative and positive cytoplasmic staining in the cells of epithelial nest. Fig.3C: H\&E stain for HBP mucosa in AuNPs + Laser treated group showing epithelium with drop-shaped rete ridges. Notice, subepithelial invasion of well differentiated SCC nests. Fig.3D: Expression of caspase 9 in AuNPs + Laser treated group showing positive cytoplasmic staining in the cells of epithelial nest.

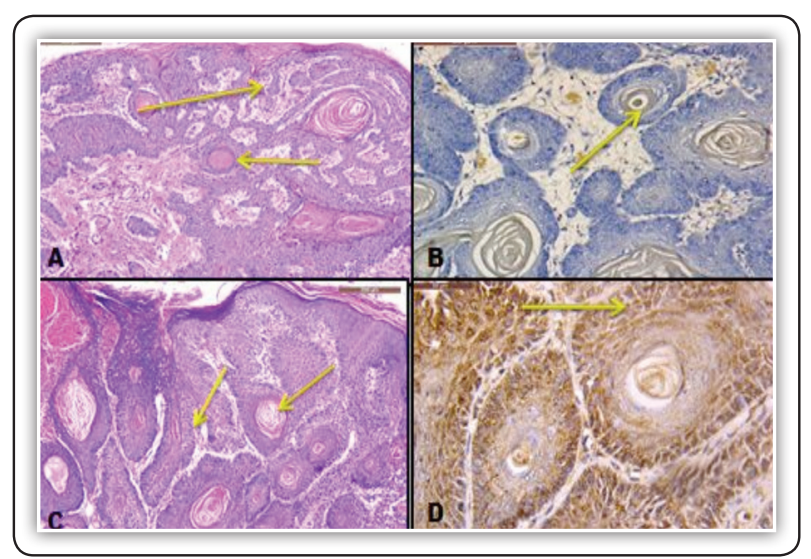

Fig.4A: H\&E stain for HBP mucosa in cisplatin + Laser treated group showing epithelium with drop-shaped rete ridges. Subepithelial invasion of well differentiated SCC nests was seen. Fig.4B: Expression of caspase 9 in cisplatin + Laser treated group showing variable immunostaining including negative and positive cytoplas mic staining in the cells of epithelial nest. Fig.4C: H\&E stain for HBP mucosa in cisplatin conjugated AuNPs + Laser treated group showing epithelium with drop-shaped rete ridges. Subepithelial invasion of well differentiated SCC nests was seen. Fig.4D: Expression of caspase 9 in cisplatin conjugated AuNPs + Laser treated group showing positive cytoplasmic staining in the cells of epithelial nest. 


\section{Image analysis and statistical analysis results:}

Correlation of the results of different groups with each other's was obtained by comparing the percentage of area distribution of caspase 9 expression in the groups used. Statistical analysis results revealed that group B5 (DMBA+ AuNPs conjugated with cisplatin + laser group) has recorded the highest mean area percentage (59.09\%), while group B1 (DMBA group) had the lowest mean area percentage (4.38\%) (Fig. 5).

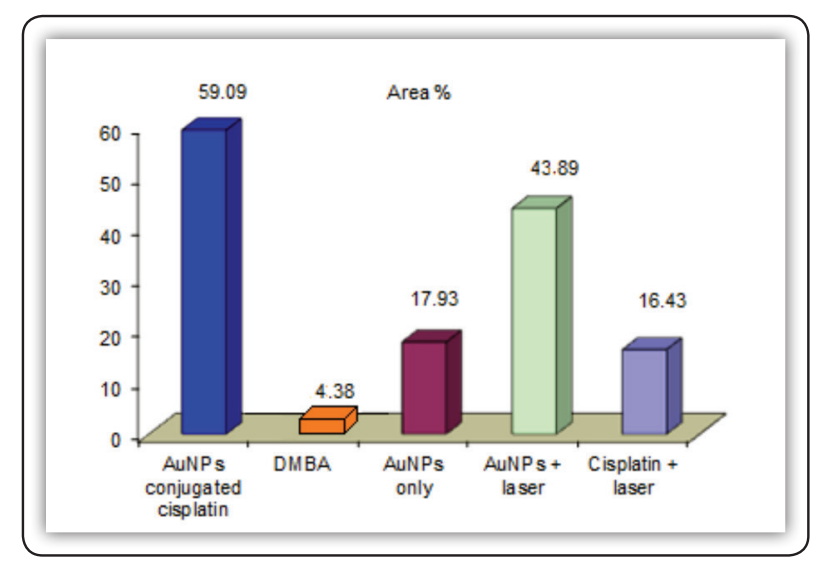

FIG.5: Bar chart representing mean area $\%$ values of caspase 9 expression among treated groups.

Comparing the results of different treated groups with (DMBA group), there was significant difference between DMBA group and the other groups. There was significant difference between (AuNPs conjugated with cisplatin + laser group) and (AuNPs group) $\{$ P value: $0.002(<0.01)\}$. There was significant difference between (AuNPs conjugated with cisplatin + laser group) and (cisplatin + laser group) $\{\mathrm{P}$ value: $0.002(<0.01)\}$. There was no significant difference between (AuNPs conjugated with cisplatin + laser group) and (AuNPs + laser group) $\{\mathrm{P}$ value: $0.245(>0.05)\}$. There was no significant difference between (AuNPs conjugated with cisplatin + laser group) and (AuNPs + laser group) $\{\mathrm{P}$ value: $0.245(>0.05)\}$.

\section{DISCUSSION}

The current results with those of other studies $^{(2,3,16)}$ support the concept that DMBA induced HBP carcinoma appears to go through the same changes as in human. Many of the structural alterations observed in carcinogen-treated HBP mucosa, both at gross and the light microscopic observations closely resemble those observed during the course of human oral cancer development ${ }^{(16)}$. AuNPs were used in this work due to its ability to cross cellular membranes, when used as a drug carrier in the medical field ${ }^{21}$. (Based on its ease of fabrication, AuNPs have a high surface area and binding capacity to target cancer cells is higher than other nanoparticles ${ }^{(22)}$. The use of AuNPs as anticancer agent was attempted by several ways as antibody conjugation ${ }^{(23)}$, intra-tumor injection ${ }^{(16)}$ and even when painted topically alone ${ }^{(24)}$.

Combinatorial chemotherapy coupled with nanomedicine have opened appealing window to the current therapeutic approaches that always failed due to tumor cell resistance and unwanted side effects of drugs on normal cells, however a great advance made when nano-based drug delivery systems paired with combination chemotherapeutic agents ${ }^{(25,26)}$. In the present study the result of group B5 (DMBA+cisplatin+ AuNPs + laser treated group) showed noticeable variation in the distribution of tumor masses and tumor size regression when compared to result that seen in group B1. The strong positive expression (50.09\%) of caspase 9 revealed that there is highly increasing in the level of cells undergone programed cell death this is in general is consistence with those of Shu et al (2014) ${ }^{(27)}$. The result obtained from this group supports that the new strategy is to improve cancer therapy by targeting tumor cell repopulation by either the AuNPs action on tumor cells in presence of laser or using the AuNPs as vehicle for addition of cytotoxic chemotherapy. Combination with PTT, immunohistochemical results revealed some differences. The positive rates of caspase 
9 protein obtained by area percentage measurements in groups B1, B2, B3, B4 and B5 were $(4.38 \%$, $17.93 \%, 43.89 \%, 16.43 \%$ and $59.09 \%$ ), respectively.

The results of the present study in groups used indicated those subjected to cisplatin+ laser only, or those injected by AuNPs only neither showed decrease in tumor size, nor positive caspase 9 immunoexpression. On the contrary, increased caspase 9 immunoexpression in AuNPs + laser group and cisplatin+ AuNPs + laser group only, which consider as an apoptotic indicator was strongly noted. This finding highly indicates improvement of cytotoxic activity of in conjugation strategy and the necessity of coupling the injection of nanoparticles and laser to achieve the favorable results of PTT in agreement with Shi et al (2013) ${ }^{(28)}$.

\section{REFERENCES}

1. Ferlay J, Isabelle S, Rajesh D, Sultan E, Colin M, Marise $\mathrm{R}$, et al. Cancer incidence and mortality worldwide: sources, methods and major patterns in globocan. Int $\mathrm{J}$ Cancer 2015; 136: 359-86.

2. Rajasekaran D, Manoharan S, Prabhakar M, Manimaran A. Nicostemma littorale prevents tumor formation in 7, 12 dimethylbenz(a)anthracene-induced hamster buccal pouch carcinogenesis. Hum Exp Tox 2015; 34: 911-21.

3. Xue T, Hua Y,Yang k, Chen D, Wang Q, Hong T, et al. Circadian variations of clock gene Per2 and cell cycle genes in different stages of carcinogenesis in golden hamster buccal mucosa. Sci Rep 2015; 5: 97-108.

4. Jiao J, Huang L, Ye F, Shi M, Cheng X, Wang X, et al. Cyclin D1 affects epithelial-mesenchymal transition in epithelial ovarian cancer stem cell-like cells. Onc Targets Ther 2013; 6:667-77.

5. Muzio L, Sartini D, Santarelli A, Rocchetti R, Morganti $\mathrm{S}$, Pozzi V, et al. Expression and prognostic significance of apoptotic genes in oral squamous cell carcinoma. Mol Carcinog 2012; 21: 45-52.

6. Vibha D, Hemlata T, Lakhan S: Comparison of cisplatinbased combination chemotherapy with carboplatin-based combination chemotherapy in oral and pharyngeal cancers. Int J Med Sci Public Health. 2016; 5: 497-99.

7. Yang C, Wang Y, Chun H, Yuan Z, Liu X, Yang F, et al.: IER5 promotes irradiation- and cisplatin- induced apoptosis in human hepatocellular carcinoma cells. Am Trans Res 2016; 8:1789-98.

8. Chukwuemeka V, Michael T, Steven J, Daniel J, Victoria B: Treatment of locally recurrent and metastatic squamous cell carcinoma of head and neck. Head and Neck Cancer Res 2016; 1: 4-9.

9. Ronald P, Raghu K. Tadagavadi, Ganesan R, William B: mechanisms of cisplatin nephrotoxicity. Toxins 2010; 2: 2491-518.

10. Florea A, Büsselberg D: Cisplatin as an anti-tumor drug: Cellular mechanisms of activity, drug resistance and induced side effects. Cancers Basel 2011; 3: 1351-71.

11. Dasari S, Tchounwou P: Cisplatin in cancer therapy molecular mechanisms of action. Eur Pharmacol 2014; 740: 364-78.

12. Kuwahara D, Tsutsumi K, Kobayashi T, Tomoko H, Nishioka K: Caspase-9 regulates cisplatin-induced apoptosis in human head and neck squamous cell carcinoma cells Cancer Lett 2000; 148: 65-71.

13. Jung AC, Ray A, Ramolu L, Macabre C, Simon F, Noulet F, et al.: Caveolin-1-negative head and neck squamous cell carcinoma primary tumors display increased epithelial to mesenchymal transition and prometastatic properties. Oncol Target 2015; 39: 184-901.

14. Masthan K: Nanotechnology-application in oral cancer. J Clin Diag Res 2014; 7: 1328-30.

15. Jain S, Hirst D, Sullivan J: Gold nanoparticles as novel agents for cancer therapy. Br J Radiol 2012; 85:101-13.

16. Afifi M, El Sheikh S, Abdelsalam M, Ramadan H, Omar $\mathrm{T}$, El Tantawi M, et al. Therapeutic efficacy of plasmonicphotothermal nanoparticles in hamster buccal pouch carcinoma. Oral Surg Oral Med Oral Pathol Oral Radiol 2013; 115: 743-51.

17. Xingjie W, Yanqin G, Chang M: Polymer/gold hybrid nanoparticles: from synthesis to cancer theranostic applications. RSC Adv 2015; 5: 787-96.

18. Kempen P, Greasley S, Parker K, Campbell J, Chang H, Jones J, et al.: Theranostic mesoporous silica nanoparticles biodegrade after pro-Survival drug delivery and ultrasound magnetic resonance imaging of stem cells. Theranostics 2015; 5:631-42.

19. Ralf P, Marina P, Iwona C, Stefan L, Christina J, Christoph A: Novel nanoparticulate drug delivery Systems. Nanomedicine Lond 2016; 11: 573-76. 
20. Amita N, Abhiney P, Rakhi G, Rajat N, Alisha S, Megha M. Comparison of immunohistochemical expression of antiapoptotic protein survivin in normal oral mucosa, oral leukoplakia, and oral squamous cell carcinoma. Pathol Res 2015; 20:793-99.

21. 21. Patra C, Bhattacharya R, Mukhopadhyay D, Mukherjee P: Fabrication of gold nanoparticles for targeted therapy in pancreatic cancer. Adv Drug Deliv Rev. 2010; 62: 346-61.

22. Mahmoud R, Bayoumi D: Use of colloidal gold nanoparticles on initiated oral dysplasia of syrian golden hamster pouches. Egy Dent 2015; 61: 665-75.

23. Jelic S, Sotiropoulos G: ESMO guidelines working group. Hepatocellular carcinoma ESMO clinical practice guidelines for diagnosis, treatment and follow-up. Ann Oncol 2010; 21: 59-64
24. Shamia A: Efficacy of nanogold conjugated cetuximab on hamster buccal pouch carcinoma. M thesis, 2015; Al Azhar University: Cairo, Egypt

25. Liboiron B, Mayer L: Nanoscale particulate systems for multidrug delivery: towards improved combination chemotherapy. Ther Deliv.2014; 5: 149-71.

26. Loro L, Vintermyr O, Johannessen A: Cell death regulation in oral squamous cell carcinoma: methodological considerations and clinical significance. Oral Pathol Med 2003; 32: 125-38.

27. Shu C, Shou Y, Cheng Y, Yeh L, Chen N: Thymoquinone induces cell death in human squamous carcinoma cells via caspase activation-dependent apoptosis and LC3-II activation-dependent autophagy. PLoS One 2014; 9:101-79.

28. Shi X, Gong H, Li Y, Wang C, Cheng L, Liu Z: Graphenebased magnetic plasmonic nanocomposite for dual bioimaging and photothermal therapy. Biomat. 2013; 34:786-93. 
